The distribution of medicated salt is actually a variant of mass drug administration, applicable in conditions when residual spraying is not, for various reasons, feasible. Chloroquine or pyrimethamine has been used for admixture with salt, but the latter drug is no longer advocated for this purpose.

(4) Radical treatment of relapsing malaria means generally the use of 8-aminoquinolines for infections due to Plasmodium vivax and $P$. malariae, since that due to $P$. falciparum is cured easily by $a$ standard three-day treatment with chloroquine or amodiaquine. The proper administration of daily primaquine or quinocide over a fourteen-day period is often difficult without a wide network of medical services, and in such cases large single doses or smaller repeated doses of a sporontocidal drug (pyrimethamine) have been tentatively given.

The following aspects of chemotherapy are among those of immediate interest for malaria eradication. There is a great difference between the approach of the clinician interested in treatment of a case of malaria and that of the malariologist whose aim is the eradication of this infection from a large community. From the point of view of the clinician no new anti-malarials are really necessary; he has quinine or, preferably, one or other of the 4-aminoquinolines for treatment of severe infections; if he wants to prevent relapses one of the modern 8-aminoquinolines-safe when given with some elementary precautions and almost 100 per cent reliable-is available; for protection from infection the clinician has the choice of proguanil, pyrimethamine, and (in the second place) chloroquine or amodiaquine; any of these drugs will serve his purpose when given at proper and regular intervals.
For the malariologist, each of these drugs has one flaw : it must be given in regular, repeated doses to achieve its effect on the transmission of malaria. In this respect they are often a failure when used on a large scale in countries without extensive and wellorganized medical and public health systems. In reassessing the problems of chemotherapy in malaria eradication two questions may be asked: How can the best use be made of the anti-malarials now avail. able?, and, What new drugs are needed, if any ?.

Improvements in operational conditions, better co-operation from an enlightened community, or the introduction of some relatively simple technical innovations may remove a number of obstacles to a wider use of drugs in malaria eradication, but are not always feasible in under-developed areas with inadequately organized public health services.

What now drugs are needed? The answer is that at least two types of anti-malarials must be developed: (1) A potent and safe schizontocidal drug which will maintain its effect for 3-6 months after a single dose. It would be advantageous if this drug had a rapid and prolonged sporontocidal action. (2) A good and safe anti-relapse drug capable of effecting a radical cure of vivax and malariae infections when given in a single dose or, at the most, in a three-day course of treatment. Over the past five years the development of new anti-malarial drugs has not been so fast or so successful as expected.

There are two reasons for the apparent lack of drive in research on new anti-malarials: one is the empirical approach to the investigation of new compounds and the other is the growing difficulty of gauging their value in human malaria; little is known about the metabolism of the infected cell.

\title{
PHYSICAL ANTHROPOLOGY OF AUSTRALIAN ABORIGINES
}

CINCE 1951 the Department of Anatomy of the $S$ University of Adelaide has sent out seven field expeditions to study the physical anthropology of the aborigines in different parts of Australia, and the first complete trans-continental survey-from Fowler's Bay (Yalata) on the south coast to the Liverpool River in northern Arnhem Land has now been compiled.

The survey covered most metrical and nonmetrical characters. Much of the information collected has yet to be analysed statistically, but some features are emerging to warrant preliminary consideration. These have been reviewed by Prof. A. A. Abbie of the Department of Anatomy, University of Adelaide, South Australia*.

Divergent opinions on whether the aborigines are homogeneous or heterogeneous lend interest to a comparison of some physical measurements of three aboriginal groups, separated from each other by some 800 miles of territory and all living in different environments.

The groups are, respectively, (a) a composite southern group assembled near Yalata on the south coast ; $(b)$ two neighbouring tribes of central Australia - the Pintubi and the Wailbrai : and $(c)$ a tribe near the mouth of the Liverpool River in northern Arnhem Land, the Burera. In the present paper only adult males are considered as being most representative

*Australian Journal of Science, 28, No. 7 (January 1961). of their groups. The numbers examined-Yalata, 33 ; Pintubi, 38 ; Wailbrai, 30 ; Burera, 26-are about the maximum obtainable in any tribe in a reasonable time. All except the Yalata group had come under European influence only shortly before the time of examination.

The figures indicate that, right across the continent, through a wide diversity of environments, the aborigines exhibit an extraordinary degree of physical homogeneity.

Up to the age of about 5 years, the physical proportions of aboriginal children are about the same as those of comparable Europeans. Between 5 and 6 years, however, aboriginal children suddenly show a marked extension of the inferior extremities that reduces the relative sitting height to about 50 per cent - close to the proportions of European children of around 12 years. Thereafter, until the cessation of growth, the aborigines preserve their six years' advantage and finish with a relative sitting height close to 46 per cent.

So far this has been worked out fully only for the central Australian males, but a preliminary survey of the data indicates that it holds for both sexes and for most northern and most southern groups as well.

New-born aborigines are pinkish-yellow with some darker colouring around the nipples, navel, genitalia and buttocks. The rate of darkening of the skin seems to depend on the degree of exposure to the 
Sun and full pigmentation may be acquired in one week or not for two or three weeks. The oral mucosa may be patchily pigmented in infancy, but usually not until eruption of the first permanent molars. The darker colouring starts around the anterior gum margins and spreads to the general mucosa and the palate with advancing maturity. There is a tendency towards fading in old age. In tribes that practise circumeision, at the time of operation (about puberty) the glans penis is pink or only slightly pigmented; in adult circumcized males the glans is as dark as the surrounding skin.

In most aborigines the skin is reddish-brown or chocolate, but more intense exposure-as in the desert or far north-produces a much darker colour. Those who wear clothes regularly tend to lose some of their melanin and fade, sometimes to near the original pinkish-yellow in parts. Such people are liable to sunburn on unaccustomed exposure.

Head hair is usually dark brown to black, but blondness-ranging from quite flaxen to reddishbrown-is common in the children of central and south Australia. The fine hair of the body is also fair, but all other aspects of pigmentation-skin, eyes, eyebrows and lashes-are as in all other aborigines. In boys the scalp hair begins to darken at 8-10 years and becomes black or dark brown, like the hair of axillæ, pubes and beard, before the age of twenty. Girls start to darken later-in adolescence- and even when adult may still only be mid-brown on the scalp although axillary hair and pubic hair is dark brown or black. In the far north light scalp hair is less common, but it occurs, and usually with a redder tinge. Light body hair is quite common there in women and children, however. This blondness probably has a genetic basis; it is certainly not due to kwashiorkor, or other nutritional disturbance.

Aborigines living under native conditions have relatively low blood pressures. In the western desert men at 40 years averaged $75-109 \mathrm{~mm}$. mercury and women slightly less. At Government settlements, where living is easier and some European food is available, male pressures were $79-119$, fernale 76-110. In a more or less urban environment male pressures averaged 89-131 (very similar to European males under similar conditions), female 75-115. In northern Arnhem Land pressures expressed on the same basis were : males 84-116, female 82-113.

All aborigines so far examined have high serum protein-levels, especially in the $\gamma$-globulin range. Serum cholesterol-levels are comparatively low in desert-living aborigines and there does not appear to be any rise with age as occurs in Europeans. Aboriginal cholesterol-levels rise with increasing Europeanization (roughly paralleling the rise in blood pressures), but not, so far, to European-levels. Serum phospholipid and mucoprotein-levels also rise under the same conditions.

\section{WEATHER FORECASTING BY NUMERICAL METHODS}

$\mathrm{T}$ HE advent of the electronic computer has made it possible to attempt weather prediction by numerical processes. The idea is to observe an initial state of the atmosphere, apply Nowton's laws and the laws of conservation of energy and mass, together with the appropriate gas laws, and hence predict the condition of the atmosphere at some time in the future, rather as the motions of heavenly bodies are predicted in astronomy. The earliest attempt was made by L. F. Richardson between the World Wars and is described in his classic book. Richardson visualized an army of computers, just able to keep up with the weather. He did not visualize computing machines, and their widespread use has changed the position, for an electronic computer can get ahead of the weather quite easily.

Active work on this subject is now proceeding in the United Kindom, Belgium, Holland, Norway, Sweden, Japan, and several centres in the United States. A recent publication from the Meteorological Office* describes some of the work being done in the forecasting research division. This paper, considered together with recent work in the United States deserves special attention because, for upper-wind forecasting, numerical methods now surpass conventional methods of forecasting. This does not mean that the conventional forecaster with his weathermaps will disappear, because, as yet, it is only the winds which are forecast numerically, not the equally important weather. A housewife may like to know that Monday will be windy because this will facilitate

* Air Ministry: Meteorological Office. Scientific Paper No. 5: An Experiment in Numerical Forecasting. By E. Knighting, G. A (London: H.M. Stationery Office, 1961.) 5 s. net. drying clothes, but she is more interested in whether or not it will rain. Numerical methods at present either do not attempt to predict this or they do so very badly.

It is obvious that the atmosphere is a very complicated affair, yet the primitive (partial differential) equations expressing the laws mentioned above look beguilingly simple. They do, however, include all the motions of the atmosphere, the steady trade winds on the surface, the great jet-streams in the upper air, depressions and anticyclones, tornadoes and showers, to mention only a few of the varied phenomena. Almost always the first thing, therefore, is to simplify the equations so that only events significant to the scale of weather concerned remain. The resulting simplified system of equations and the somewhat artificial atmosphere it describes then become a 'model'.

In the United States experience is that the simplest and earliest model-the barotropic model-remains the best for forecasting large-scale phenomena. After pioneer work by J. Charney, the great labour of extensive trials has been borne by a joint numerical weather prediction group at Suitland, Maryland. The model regards the atmosphere as describable in every way by events at one level only, and the resulting simplified atmosphere is called the equivalent barotropic atmosphere. By extending the calculation to include almost the whole northern hemisphere, and by correcting artificially for the unexplained incorrect backward motion predicted for the phenomena of the largest scale, a useful extra skill above the conventional forecaster is obtained and, for long-range air navigation, winds forecast in this way are now used in the United States. 